\title{
Energy Management with Disaster Intimation and Control using IoT
}

\author{
Mallikarjun Mudda \\ Assistant Professor, Dept. of E \&C Engineering \\ Jain University, Bangalore, India
}

\author{
Bandi Madhu Sowmya, P Lalitha Ram sai, Alluri Uday \\ Veena, Tadikamalla sai vikas \\ Dept. of E \& C Engineering, SET- Jain University \\ Bangalore, India
}

\begin{abstract}
In the area of digitization and automation, the life of human being is getting simpler as almost everything is automated. Nowadays humans have made internet an integral part of their everyday life without which they are helpless. Internet of things (IoT) gives a platform which allows different devices to inter-connect, sense and control the things remotely across a network infrastructure without any limitation to the coverage area. In our proposed work, we stress on WirelessHome-Automation-System (WHAS) using IoT, it is a system uses computers or smart phone to control basic home functions and features automatically through internet from anywhere around the world, an automated home is sometimes called a smart home. The proposed system is able to monitor the entire things connected to the internet and also to be maintaining the status of individual devices for further action. We have built the home automation with several devices and sensors, here sensor help to monitor the device status and intimate the authorized person to take particular action.
\end{abstract}

Keywords-Home Automation System, IoT, data storage, Raspberry Pi 3B, Web monitoring, Security credential

\section{INTRODUCTION}

The internet on things is the most upcoming popular application used and adopted by worldwide. In this application the authorized user can access the data and acknowledge on the particular things from irrespective of place, the internet on things made a human life much easier way to handle the things or devices from anywhere with internet on smart phone or personal computer. The system should be able to encrypt the authorized credential and protect from the third party attacks, The internetworking of physical devices, vehicles, buildings and other items embedded with electronics, software, sensors, and network connectivity that enable these devices to collect and exchange data. Internet on things leads to better and comfort on energy management as it will be able to control the device status and take action as per desired outcome. The Energy will be inexorably linked to the internet on things. The many number of devices, industries, buildings, transport systems and smart cities are connected and controlled through the Internet.

\subsection{Need for Automation}

Enough progress in home automation technology occurred to make it worthwhile to "smarten" up our home. The purpose of being able to control your security system from afar and cut your energy costs. Ultimately, at some point, our various home appliances could be connected, allowing us to control them all from our phones or another type of device. For now, some appliances can be controlled by using a Wi-Fi Connection, but you need separate smart phone apps or devices to tell them what to do. We won't even need to touch a button (for instance, you could simply walk into a room and your curtains would open, the temperature would turn up, and your favorite song would start playing). Ultimately, if it's set up with compatible devices and connect with one software platform, one could have a controlled, "smart" environment. Some home security systems are getting pretty close, giving users the ability to control temperatures and see who knocks at their door when they're away from home Tying Security cameras into home automation system allow to use an app or other sources to see when a visitor arrives, whether it's an actual delivery person or a thief posing as a delivery person. By using the home automation systems with a front door camera, one can see any visitors and respond to them audibly, making it appear as though we're home even when we are many miles away.

\section{RELATED WORK}

Work [1] proposes an Advanced Home Automation system using Open source Android, in this review paper many technologies for implementing Home automation systems were discussed and compared too. The Method used to implement and Limitations of the respective Home Automation System are discussed. "IoT based Interactive industrial Home wireless system, Energy Management System and embedded data acquisition system to display on webpage using GPRS,SMS and E-mail alert", In this paper the microcontroller ARM LPC2148 is used to implement the hardware and data acquisition. UART terminals UART0 interfaced with Zigbee module and UART1 with GSM module [2]. Several sensors are interfaced to the processor along with the LCD, for the data 
read by the sensors all the time. The data is stored in the flash memory of the processor. Flash memory interfaced to the processor through SPI protocol. The measured values are transmitted to the control unit via Zigbee, displayed on the computer and sent to the internet via WAN, here the drawback is using Zigbee protocol for data acquisition and the implementation cost to build the network increases with the size of network. [3] A Home Automation system using Internet of things to control household appliances remotely. The microcontroller used in this work is Intel Galileo. Here all the devices connected to the internet are continuously monitored and the data is saved on the cloud. The webpage is built using the HTML language and cloud computing is practiced. [4] In this work proposes Enabling IoT services for Wifi-Zigbee gateway for Home automation. This system uses Cubietruck board interfaced with wifi module (gateway) and uses TCP/IP communication protocols to enable internet. Cubietruck transmits sensor data to wifi through communication protocols. The gateway provides methods to access the sensors for user interaction and bridges interconnection between different protocols.

This primary objective of this paper is to design a Home Automation system that employs the integration of multi-touch mobile devices, cloud networking, wireless communication, and power-line communication to provide the user with remote control of various lights and appliances within their home. This system uses a consolidation of a mobile phone application, handheld wireless remote, and PC based program to provide a means of user interface to the consumer [5].

\section{SYSTEM ANALYSIS}

\subsection{Problem Definition}

Energy Management is an increasingly critical focus area for utilities and energy service providers, as well as end customers. Energy consumption needs to be minimized without compromising on comfort and other ergonomic considerations. An efficient energy management system helps optimize energy consumption. An advanced energy management system can ensure monitoring of building conditions, equipment status, utility sub-metering. Such system can also focus on maintenance (remote operation and control of equipment).

\subsection{Objectives}

The main objective is to design and implement a system using IoT, to limit the unnecessary energy flow commercially as well as in industrial level. The main challenges are high cost of ownership, deploy communication system to manage, make use of pre-installed devices to run the system.

\section{PROPOSED SYSTEM}

We will be making use of Raspberry pi $3 B$ interfaced with Pi camera module and PIR sensor for Motion Detection. Flame Sensor is also interfaced on the Raspberry PI for Detecting Disaster in households through fire. For controlling the electrical Appliances which run on AC, we make use of Relay boards. Here we are using two channel Relay Boards, converting the input working on $12 \mathrm{~V}$ DC to $240 \mathrm{~V}$ AC. To provide Raspberry $\mathrm{Pi}$ with the internet we can connect our processor to LAN through Wi-Fi or enabling internet through LAN cable or through Dongle connected to one of the USB ports. It's necessary that the processor (Raspberry $\mathrm{Pi}$ ) is always connected to the internet. Also connected to the GSM. The Emergencies are called via GSM interfaced to the Raspberry Pi.

\subsection{System Design \& Implementation}

The following figure 1 shows the block diagram of the proposed system. The sensors are used here to monitor the status and we have connected many devices through the relays and will be controlled by Raspberry pi with GSM through which the authorized user command the instruction to the system and control the device status.

\subsection{Proposed System Functions}

The Proposed system can monitor the following sensors and components:

- Motion Detection

- Fire and Smoke Detection

- The proposed System can control following appliances:

- Lights on/off

- Fans on/off

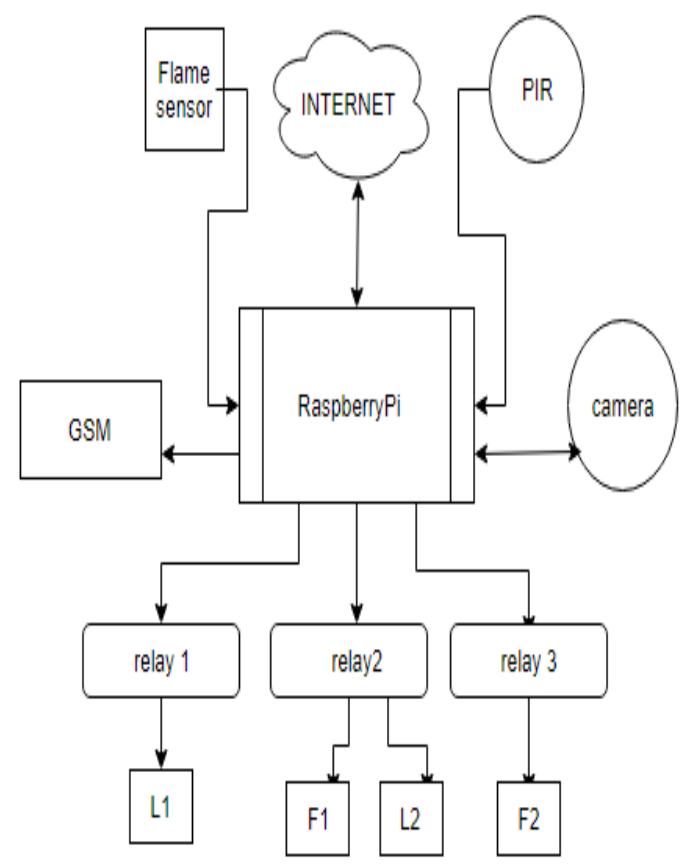

Figure 1. Proposed System Block Diagram

\subsection{Software Design}

The front end for the webpage is designed using HTML and CSS. Backend design is PHP at the server-side and Java Script at the client side. 


\subsection{System Implementation and Working}

As the main aim of the project is to optimize the energy used by the electrical appliances in households, industries, buildings, or an entire city and can be accomplished by laying an inter connection between all the devices(controllers) used in various areas to the internet which ultimately results in Internet of Things(controllers).To find out who has s been in the house, A parent detector such as the motion detection is connected to raspberry pi, when any unauthorized intrusion occurs, video triggering starts via Raspberry Pi camera module. It records the video with date and time labels (filename).Therefore; this can also be a security to the household. User's privacy concerns a lot in this system, the webpage holds the control through the pre-installed camera at the area of operation. Camera captures the images, the moment it detects the motion automatically and sends the copy of the image to the drop box. Here the drop box acts as a database or a cloud which holds the images from the camera. A flame sensor is deployed on the raspberry pi, such that if any disaster happens in the house such as Kitchen fire or short-circuits fire, an immediate call is made to the emergencies.

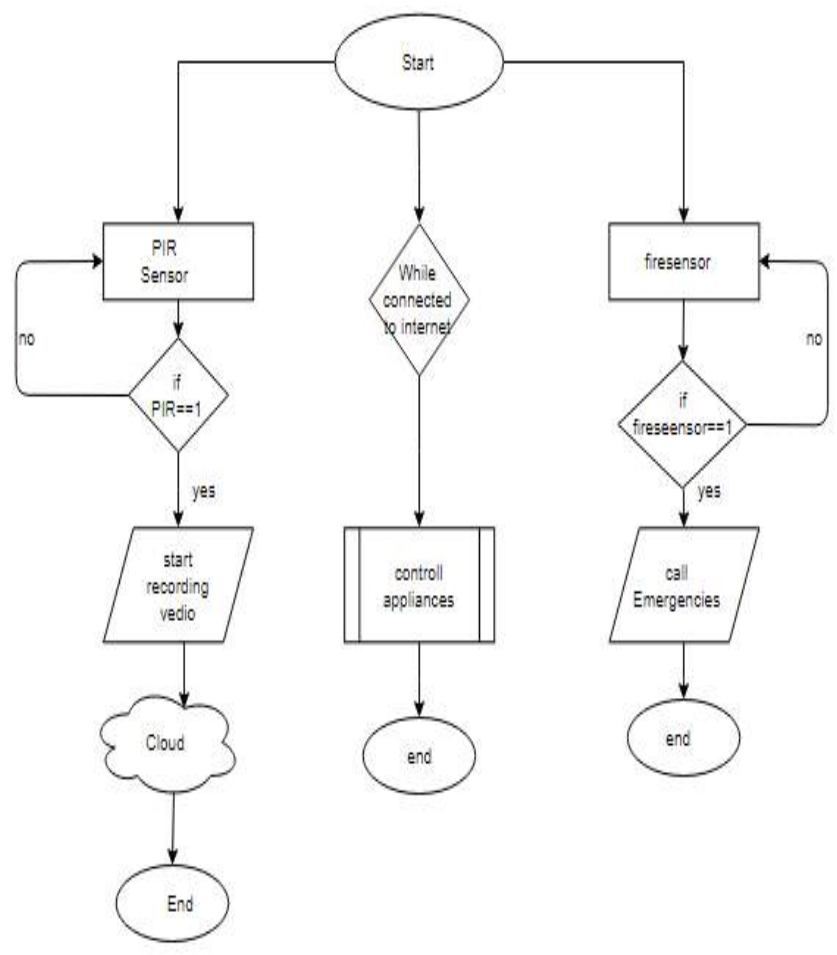

Figure 2. System flow graph

Step 1: Monitors for the intrusion, if any intrusion gets detected i.e., PIR senses change in the IR signature and turns ON the Pi camera installed on the RPi to start either recording or captures the images the moments soon after it detects the intrusion. Through GSM a notification at the end user can be sent in the form of a text message via GSM module. The captured image or video is uploaded on to the Drop box. This can be previewed by logging into the registered Drop box account. (Webpage or application)
With intrusion detection, the electrical appliances can be turned on automatically.

Step 2: Monitors for the disaster in the household, if fire explodes i.e., the module makes use of fire sensor and comparator for the change in the IR radiation from the flame. A Call will be made to emergencies, indicating there is an emergency situation and a buzzer interfaced on the raspberry pi acts as alarm, and is activated on for the fire explosion.

Step 3: While connected to the internet, the electrical appliances can be controlled remotely.

The second stage is actually the controlling stage in which all the electrical appliances can be accessed through the internet, Controlling the appliances in the household from remote place can be done just by knowing the IP address of the network to which the raspberry pi is connected to, that can be either through an android application or through a web address containing a layout of all the appliances. In order to gain the access over the layout the user has to login with a unique password. Here The IP address of the Raspberry Pi can be held fixed by port forwarding.

\section{RESUlTS \& DisCUSSION}

The proposed system is shown with figure 3 , the number of sensors and devices like: speaker, motion detector, light etc.

Interface of PIR sensor and camera module on RaspberryPi is shown in Fig.13. The PIR sensor which is connected will detect the motion if any and will send the input to RaspberryPi. As soon as the motion is detected the camera gets active and will capture the image and video of the intrusion. This will be stored in the Raspberry Pi and also will be sent to Drop box.

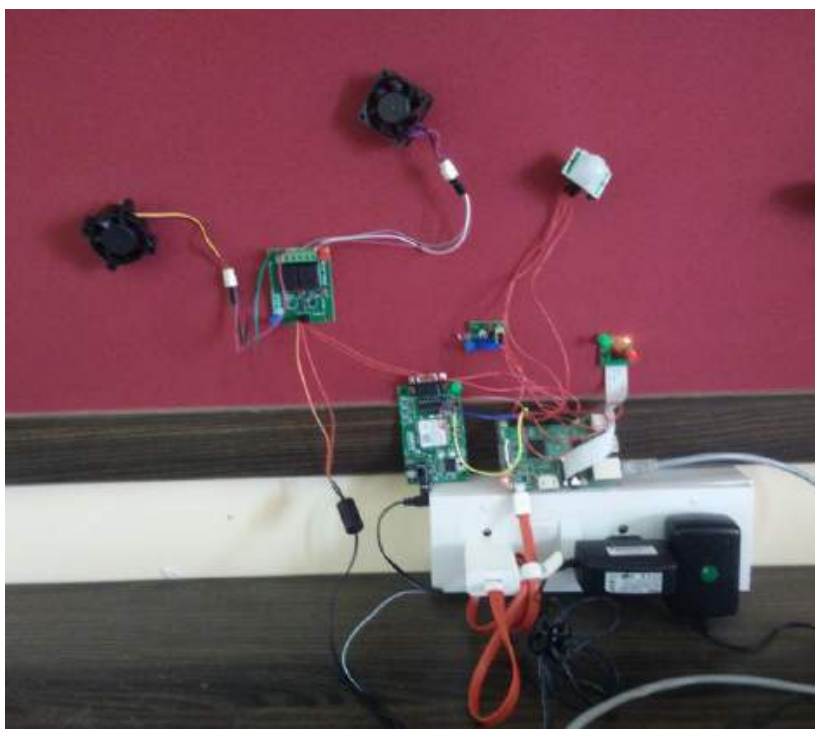

Figure 3. Entire setup of the sensors and the processor.

The code written in python script is simulated on the terminal before burning the code on the processor, and when the motion is detected the following figure 4 shows the 
terminal output and the same captured video or the image is uploaded to the respective cloud the figure 5. Illustrates the Drop box upload from the pi, with date and time mentioned.

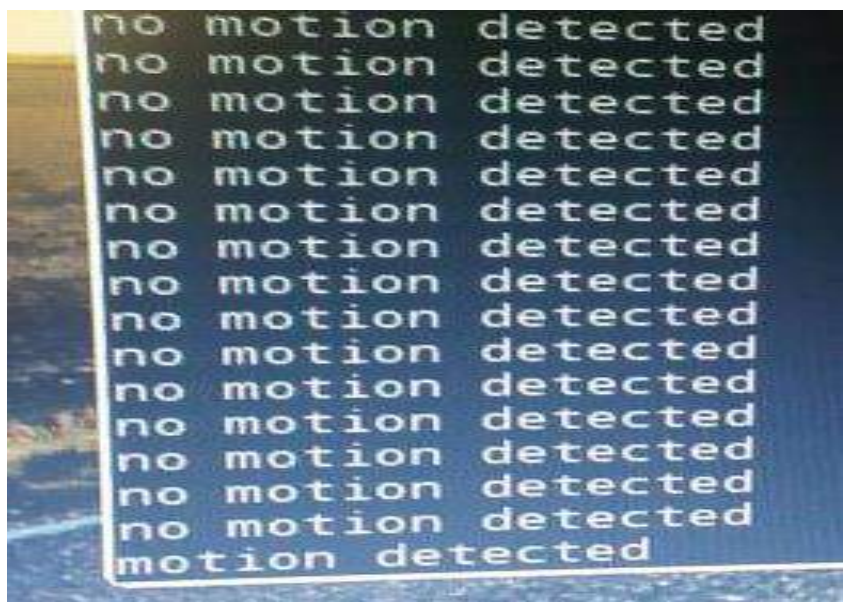

Figure 4. Motion Detection

\begin{tabular}{|c|c|c|}
\hline 9 & $6: 41$ & "प口" (c) 46 (29) \\
\hline$>$ & $\begin{array}{l}\text { Files - Dropbox } \\
\text { https://www.dropbox.com }\end{array}$ & : \\
\hline & pi & $Q$ \\
\hline
\end{tabular}

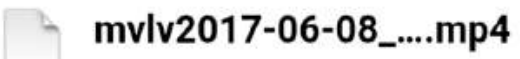

6/8/2017 9:00 AM, 75.25 KB

mvlv2017-06-08_....mp4

6/8/2017 9:00 AM, 67.42 KB

\section{mvlv2017-06-08_....mp4}

6/8/2017 9:00 AM, 86.45 KB

\section{mvlv2017-06-08_....mp4}

6/8/2017 9:00 AM, 53.07 KB

Figure 5. Drop box upload from Pi.

Similarly when the Fire explosion takes place the flame sensor reads the value high and sends the same to the processor, as the input to the processor from the sensor is high, a call is being made to the emergencies. The same is shown in Figure 6. The connections are shown in Figure 6(a) and when fire is detected the sensor reading high is shown in Figure 6(b). Interface of buzzer on RaspberryPi through which it is alarmed and Figure 6(c) shows the terminal output of fire detection.
Volume: 02, Issue: 08, ISBN: 978-0-9957075-9-7, August 2017

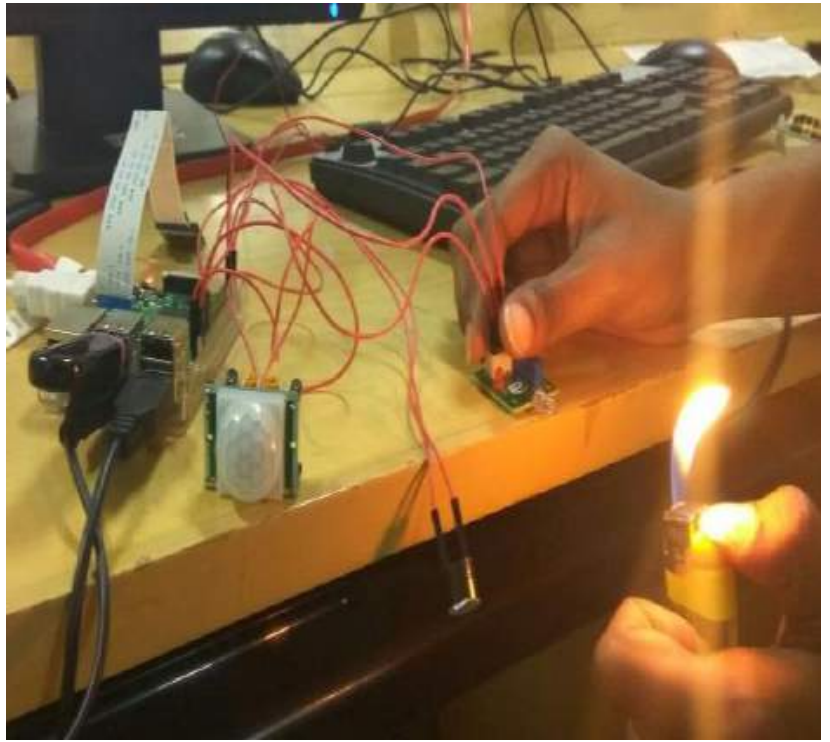

Figure 6(a). Flame sensor detecting fire and alarmed thru buzzer

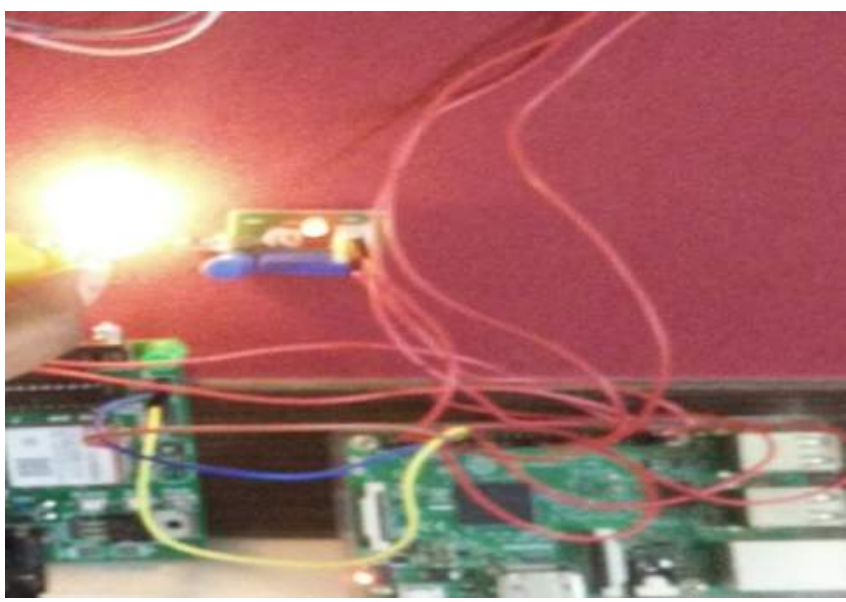

Figure 6(b). Sensor reading High When Fire Explodes.

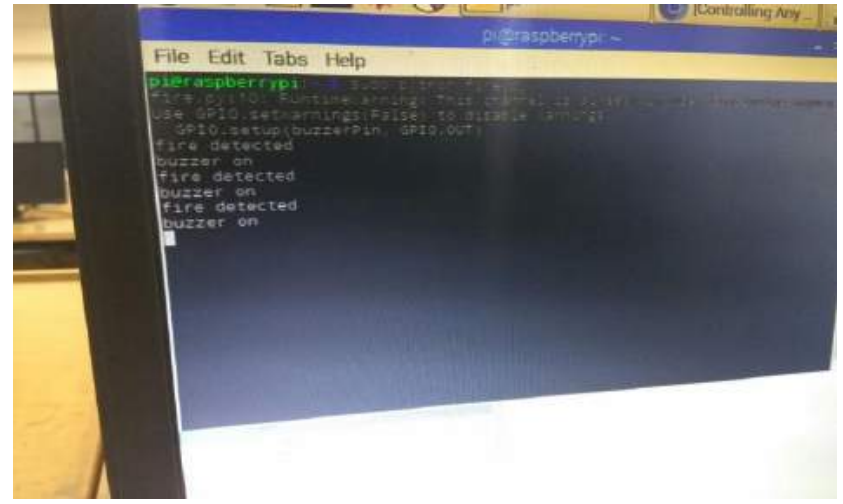

Figure 6(c).Terminal output after flame detection

When fire is detected by the sensor module, then it will write digital high output on the flame sensor. The out is read by 
RaspberryPi on pin18. Depending on the status of the pin, Buzzer is activated and GSM module will place the call.

\section{Home Automation}

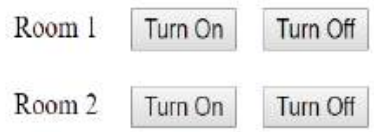

Figure 7. Webpage view for on and off.

The above figure shows the layout of the switch buttons for switching on/off electrical appliances for different rooms in a household. The webpage is created using HTML and PHP languages. The webpage contains login and password too. This varies according to the ip address of the client.

\section{CONCLUSION}

The home automation using Internet of Things has been experimentally proven to work satisfactorily by connecting simple appliances to it and the appliances were successfully controlled remotely through internet. The designed system not only monitors the sensor data, like fire, motion sensors, but also actuates a process according to the requirement, for example switching on the light when it gets dark. It also stores the sensor parameters in the cloud (Drop Box) in a timely manner. This will help the user to analyze the condition of various parameters in the home anytime anywhere. Using this system as framework, the system can be expanded to include various other options which could include home security feature like capturing the photo of a person moving around the house and storing it onto the cloud. This will reduce the data storage than using the CCTV camera which will record all the time and stores it. The system can be expanded for energy monitoring, or weather stations. This kind of a system with respective changes can be implemented in the hospitals for disable people or in industries where human invasion is impossible or dangerous, and it can also be implemented for environmental monitoring.

Automation of Turning on the cylinders, depending upon type of fire detected fixed in buildings can be made. Safety measures can be scientifically automated and should be easily operatable using advanced sensors and processors.

\section{REFERENCES}

[1] Yashodeep Patin and Prof. S.P Dhanure,"Advanced Home Automation System Using Open source Android Operated Application", International Research Journal of Engineering and Technology, Volume 03,Issue 04|,April-2016.
[2] Riyaj Kazi and Gaurav Tiwari, "IoT based Interactive industrial Home wireless system, Energy Management System and embedded data aquisition system to display on webpage using GPRS,SMS and E-mail alert", 2015 International Conference on Energy System and applications (ICESA).R. Nicole.

[3] Vinay Sagar K N and Kusuma K N, "Home Automation using Internet of Things", in International Research Journal of Engineering and Technology, Volume 02,issue 03,June 2015.

[4] Vivek G.V and Sunil M.P, "Enabling IoT Services using Wi-Fi- Zigbee Gateway for Home automation system",2015 IEEE International Conference Research in Computational Intelligence and Communication Networks (ICRCICN).

[5] Chathura Withanage, Rahul Ahok, Chau Yuen and Kevin Otto " A Comparison of the Popular Home Automation Technologies", 2014 IEEE Innovative Smart Grid Technologies - Asia (ISGT ASIA).

[6] Deepali Javale, Mohd. Mohsin, Shreerang Nandanwar "Home Automation and Security System Using Android ADK" in International Journal of Electronics Communication and Computer Technology (IJECCT) Volume 3 Issue 2 (March 2013).

[7] Basil Hamed, "Design \& Implementation of Smart House Control Using LabVIEW" at International Journal of Soft Computing and Engineering (IJSCE) ISSN: 2231-2307, Volume-1, Issue-6, January 2012.

\section{AUTHOR PROFILE}

Dr. Mallikarjun Mudda received his B.Tech degree from Electronics and Communication Engineering from Visvesvaraya Technological University, India, in 2008, and then he received M.Tech degree from Digital Communication Engineering from Visvesvaraya Technological University, India, in 2012, and he completed Ph.D. from Electronics Engineering, Jain University, Bangalore, India. He is currently a faculty member of School of Engineering and Technology, Jain University. His research interests areas are medical image processing, satellite image processing, remote sensing, wireless network security, mobile computing.

Bandi Madhu Sowmya, P Lalitha Ram sai, Alluri Uday Veena, Tadikamalla sai vikas have completed their B. Tech from school of Engineering and Technology, Jain University and working in Capgemini software company, Bangalore.

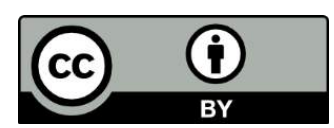

() 2017 by the author(s); licensee Empirical Research Press Ltd. United Kingdom. This is an open access article distributed under the terms and conditions of the Creative Commons by Attribution (CC-BY) license. (http://creativecommons.org/licenses/by/4.0/). 